\title{
Epidemiological study of intracranial meningiomas in a tertiary care hospital
}

\author{
Avdhesh Shukla ${ }^{1}$, Asheesh Kumar Gupta ${ }^{1}$, \\ Anand Sharma ${ }^{1}$, S. N. lyengar ${ }^{1}$ \\ 1 Department of Neurosurgery, G. R. Medical College Gwalior, INDIA
}

\begin{abstract}
Meningiomas are tumours that arise from the meningothelial cells. Most of these tumours are intracranial; some are intraspinal and few extra cranial. There are many histological variants classified into three grades depending on clinical behaviour. Classification is important for determining the modality of treatment. Objectives: To study the incidence, location, sex and age predilection, histological variants and grading of meningiomas based on WHO 2007 classification and recurrence if present. Materials and methods: All 200 cases of meningiomas. Based on Histological features, typing and grading of meningiomas was done as per the WHO 2007 classification of Meningiomas. Age, Sex incidence, Location of meningiomas were studied. Results: Meningiomas comprised $26.17 \%$ of all CNS tumours during the study period. Of 764 CNS tumours, 200 were meningiomas. Most of them were intracranial, predominantly involving the convexities of brain, females and the $41-50$ age group. Of these, 180 were benign grade I tumours, 12 were grade II and 8 were grade III. The most common histological variant was fibroblastic and meningothelial. Grade II and Grade III tumours commonly recurred. Conclusion: Meningiomas are slow growing tumours arising from the meningothelial cells accounting for $26.17 \%$ of all CNS neoplasms showing a variety of histological patterns, more common in women, predominantly Grade I tumours. Recurrence of tumours depends on histological grade and extent of surgery.
\end{abstract}

\section{INTRODUCTION}

A meningioma is a tumour that develops from the specialized meningothelial cell called as arachnoidal cap cells, the membrane that surrounds the brain and spinal cord, and located along the parasagittal sinus, over the cerebral convexity, sphenoid wing, around the pontocerebellar angle and along region of the spinal cord (1). Meningiomas constitute approximately a quarter of central nervous system (CNS) neoplasms. Most meningiomas (90\%) are categorized as benign tumours, with the remaining $10 \%$ being atypical or malignant.

Harvey Cushing in 1922 coined the name "meningioma" for the most common dural based tumour, accounting for $15-30 \%$ of all primary intracranial tumours (2). These tumours can occur in any age, but commonly present in middle age and has a female preponderance,

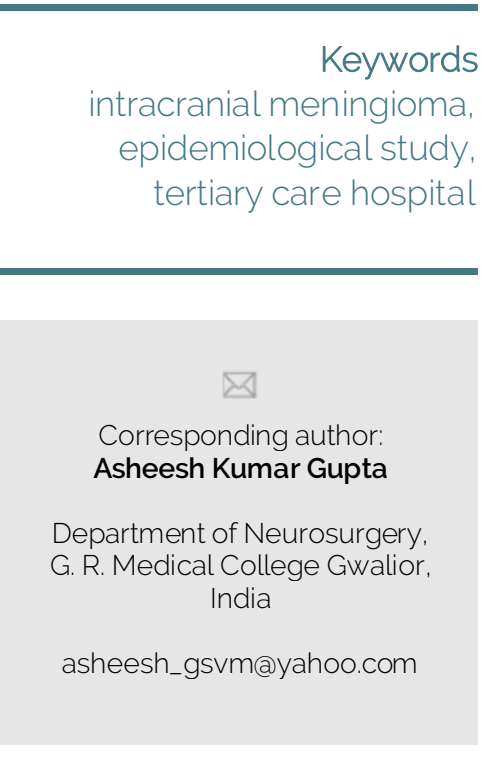

Copyright and usage. This is an Open Access article, distributed under the terms of the Creative Commons Attribution Non-Commercial No org/licenses/by-nc-nd/4.0/) which permits noncommercial re-use, distribution, and reproduction in any medium, provided the original work is unaltered and is properly cited.

The written permission of the Romanian Society of Neurosurgery must be obtained for commercial re-use or in order to create a derivative work.

ISSN online 2344-4959

(C) Romanian Society of Neurosurgery

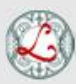

First published

March 2019 by

London Academic Publishing www.lapub.co.uk 
with a female/male ratio of approximately 2:1 intracranial and 10:1 on the spine. Genetic factors also play a role in meningioma development and predisposition. Type 2 neurofibromatosis (NF2) is an autosomal dominant condition related to a mutation on chromosome 22q12 and is a common condition related to increased risk for developing meningiomas, among other neoplasms (3). Ninety percent of meningiomas are benign, $6 \%$ are atypical, and $2 \%$ are malignant tumours (4).

Meningiomas vary in their symptoms, cranial meningiomas may cause seizures, headaches, and focal neurological deficits. Diagnosis is made by a contrast enhanced CT and/or contrast MRI (magnetic resonance imaging) scan. While MRIs are in some ways superior, the CT-scan can be helpful in determining if the tumour invades the bone, cause hyperostosis of bone.

Most patients with meningioma undergo resection to relieve neurological symptoms. Complete resection is often curative. For incompletely resected or recurrent tumors not previously irradiated, radiotherapy is administered. Two of the most important factors that determine the prognosis in patients with meningiomas are the extent of the resection and the tumor's histological grade (5). Although as a group they are considered to be benign, symptoms, variability in recurrence frequency, life expectancy, histological appearance and prognosis exist.

\section{MATERIAL AND MEthodS}

This study is a retrospective study conducted in the Department of Neurosurgery, G. R. Medical College and Jay Arogya Hospital, Gwalior, M.P. India, over a period of 5 years. Of all CNS tumours, only cases of meningiomas during the study period were included. Meningiomas in all age groups and both sexes were included in the study. Other CNS tumours were excluded. These cases were analysed for age, sex incidence, location and histopathological diagnosis. Statistical analysis was done by calculating the numbers and percentage for computing the incidence in various age groups, in sexes, location and HPE diagnosis.

Study design: A meta-analysis

Ethical approval: The study was undertaken after consent and clearance by the ethical committee of G.R. Medical College Gwalior

Inclusion criteria: Of all CNS tumours, only cases of meningiomas during the period 2012 - 2017 were included. Meningiomas in all age groups and both the sexes were included in the study.

Exclusion criteria: Other CNS tumours were excluded.

Sample size: Two hundred cases of meningiomas Methodology: Based on Histological features, typing and grading of meningiomas was done as per the WHO 2007 classification of Meningiomas. Age, Sex incidence, Location of meningiomas were studied.

Statistical analysis: It was done by calculating number and percentage for computing the incidence in various age groups, in sexes, location and also comparison with other studies.

\section{OBSERVATION AND RESULTS}

TABLE 1: Age wise distribution of patients

\begin{tabular}{|c|c|c|c|}
\hline S.No. & Age (yrs) & No. of patients & Percentage \\
\hline 1. & $<20$ & 9 & $4.5 \%$ \\
2. & $20-40$ & 75 & $37.5 \%$ \\
3. & $41-60$ & 96 & $48 \%$ \\
4. & $>60$ & 20 & $10 \%$ \\
\hline
\end{tabular}

TABLE 3: Presenting complaints

\begin{tabular}{|c|c|c|c|}
\hline S.No. & $\begin{array}{l}\text { Clinical } \\
\text { presentation }\end{array}$ & $\begin{array}{l}\text { No. of } \\
\text { patients }\end{array}$ & Percentage \\
\hline 1. & Headache & 178 & $89 \%$ \\
\hline 2. & Seizure & 96 & $48 \%$ \\
\hline 3. & Raised ICP & 80 & $40 \%$ \\
\hline 4. & Ptosis & 20 & $10 \%$ \\
\hline 5. & Hemiparesis & 69 & $34.5 \%$ \\
\hline 6. & $\begin{array}{l}\text { Behaviour } \\
\text { problem }\end{array}$ & 15 & $7.5 \%$ \\
\hline 7. & $\begin{array}{l}\text { Memory } \\
\text { difficulties }\end{array}$ & 40 & $20 \%$ \\
\hline 8. & $\begin{array}{l}\text { Visual } \\
\text { problem }\end{array}$ & 27 & $13.5 \%$ \\
\hline
\end{tabular}


TABLE 2. Gender wise distribution of patients

\begin{tabular}{|c|l|c|c|}
\hline S.No. & Gender & No. of patients & Percentage \\
\hline 1. & Male & 92 & $46 \%$ \\
2. & Female & 108 & $54 \%$ \\
\hline
\end{tabular}

TABLE 4: Distribution of patients according to location of tumour

\begin{tabular}{|c|c|c|c|}
\hline S.No. & Location of tumour & No. of patients & Percentage \\
\hline 1. & Falx or parasagital & 40 & $20 \%$ \\
\hline 2. & Convexity & 80 & $40 \%$ \\
\hline 3. & Sphenoid wing & 20 & $10 \%$ \\
\hline 4. & Olfactory groove & 13 & $6.5 \%$ \\
\hline 5. & Petroclival & 3 & $1.5 \%$ \\
\hline 6. & Posterior fossa \& CP angle & 22 & $11 \%$ \\
\hline 7. & Tentorial & 9 & $4.5 \%$ \\
\hline 8. & Pterional & 2 & $1 \%$ \\
\hline 9.. & Tuberulam sellae & 2 & $1 \%$ \\
\hline 10 & Intraventricular & 5 & $2.5 \%$ \\
\hline 11 & Diploic & 2 & $1 \%$ \\
\hline 12 & Foramen magnum & 2 & $1 \%$ \\
\hline
\end{tabular}

TABLE 5. Type of Craniotomy

\begin{tabular}{|c|c|c|}
\hline Location of tumour & No. of patients & Percentage \\
\hline Fronto-Temporo-Parietal craniotomy & 20 & $10 \%$ \\
\hline Fronto- Parietal craniotomy & 62 & $31 \%$ \\
\hline Frontal & 33 & $16.5 \%$ \\
\hline Temporo-parietal & 19 & $9.5 \%$ \\
\hline Tempro-parieto-occipital & 9 & $4.5 \%$ \\
\hline Bifrontal & 15 & $7.5 \%$ \\
\hline Parietal & 5 & $2.5 \%$ \\
\hline Sub-occipital & 24 & $12 \%$ \\
\hline Parieto-occipital & 13 & $6.5 \%$ \\
\hline
\end{tabular}


TABLE 6. Distribution of patients according to according to surgical excision

\begin{tabular}{|c|c|c|c|}
\hline S.No. & Simpson grade & No. of patients & Percentage \\
\hline 1. & I & 30 & $15 \%$ \\
2. & II & 135 & $67.5 \%$ \\
3. & III & 17 & $8.5 \%$ \\
4. & IV & 16 & $8 \%$ \\
5. & V & 2 & $1 \%$ \\
\hline
\end{tabular}

TABLE 7. Distribution of patients according to size of the tumour

\begin{tabular}{|c|c|c|c|}
\hline S.N. & Size of tumour & No. of patients & Percentage \\
\hline 1 & $1-3 \mathrm{~cm}$ & 0 & 0 \\
2 & $3-4 \mathrm{~cm}$ & 138 & 69 \\
3 & $4-5 \mathrm{~cm}$ & 42 & 21 \\
4 & $>5 \mathrm{~cm}$ & 20 & 10 \\
\hline
\end{tabular}

TABLE 8. Distribution of patients according to grade

\begin{tabular}{|c|c|c|c|}
\hline S.No. & Grade & No. of patients & Percentage \\
\hline 1. & I & 180 & $90 \%$ \\
2. & II & 12 & $6 \%$ \\
3. & III & 8 & $4 \%$ \\
\hline
\end{tabular}

TABLE 9. Post op complications

\begin{tabular}{|c|c|c|}
\hline S.No. & Post op complications & No. of patients \\
\hline 1. & Infection & 38 \\
2. & Seizure & 23 \\
3. & Hemiparasis & 86 \\
4. & Visual loss & 1 \\
5. & Behavior change & 28 \\
6. & Memory deficit & 43 \\
7. & Raised (ICP) & 13 \\
\hline
\end{tabular}


TABLE 10. Patient follow up data given as frequency

\begin{tabular}{|c|c|c|c|}
\hline S.No. & Follow up & $\begin{array}{c}\text { No. of patients } \\
\text { with recurrence }\end{array}$ & $\%$ \\
\hline 1. & $<2$ year & 5 & $2.5 \%$ \\
2. & $2-5$ years & 12 & $6 \%$ \\
\hline
\end{tabular}

FIGURE 1. Pre op and post op CT of tuberculum sellae meningioma. FIGURE 2. Pre op and post op CT of olfactory groove
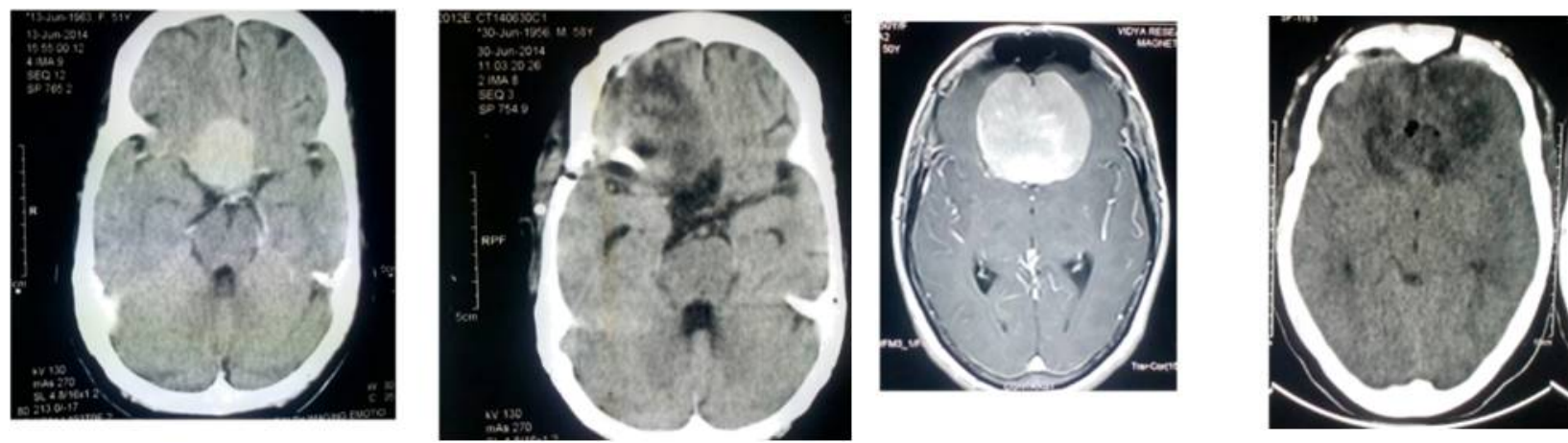

FIGURE 3. Pre op and post op CT of posterior fossa meningioma

FIGURE 4. Pre op and post op CT of sphenoid wing meningioma
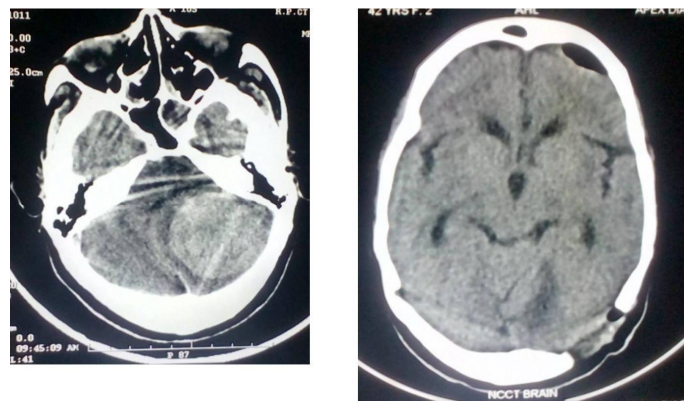

FIGURE 5. Pre op and post op CT of frontal convexity meningioma
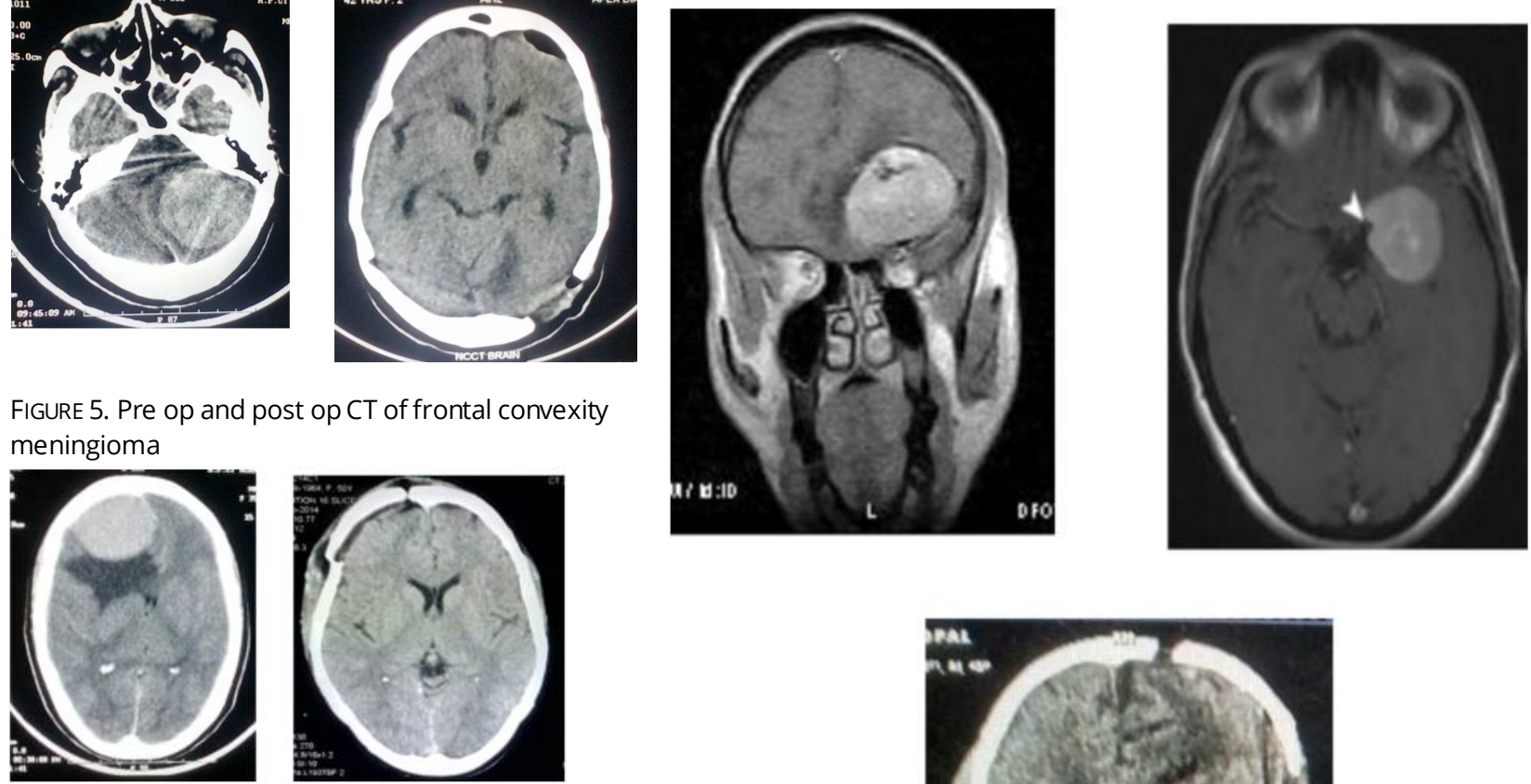

FIGURE 6. Pre op and post op CT of para saggital meningioma
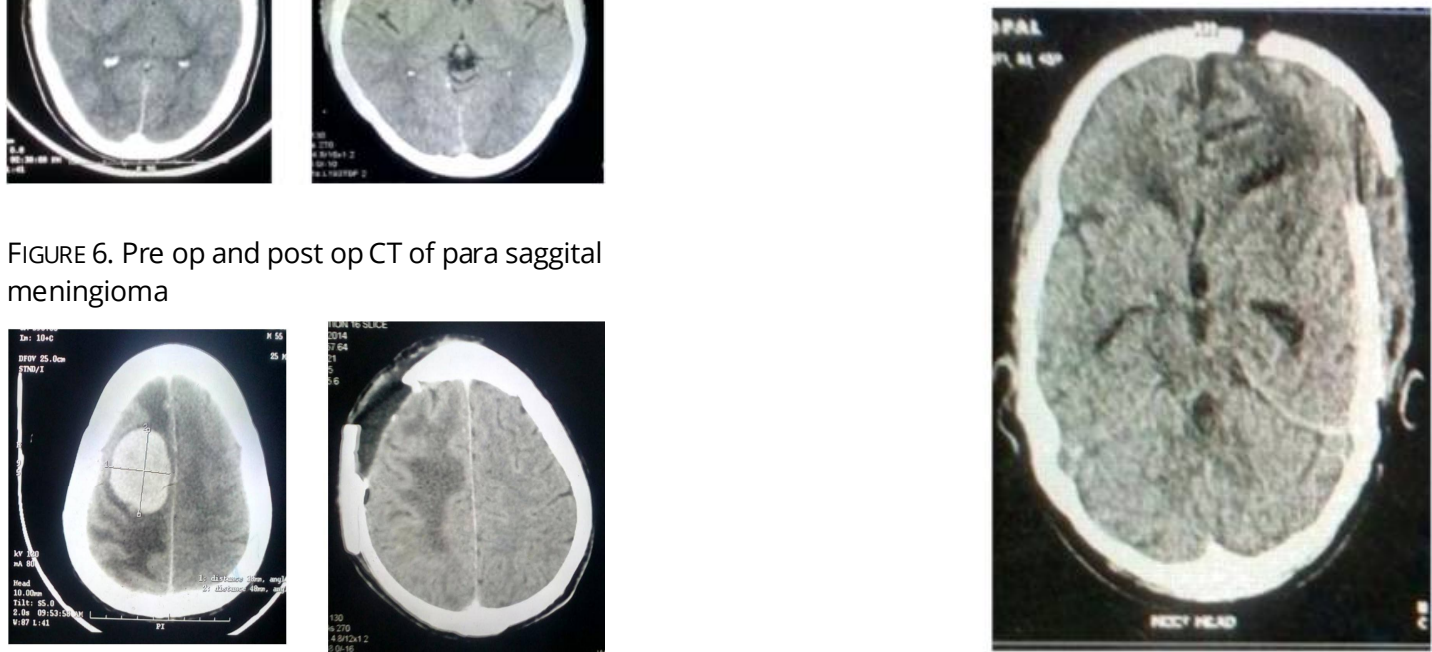


\section{DISCUSSION}

Meningiomas constitute 25 - 30\% of all CNS tumours and are the most common tumour arising from the meninges (6). In our centre out of 764 cases of CNS tumours, Meningiomas constituted 200 (26.17\%), similar to studies by AB, Shah et al (7), Ruberti R F (8), Intisar SH Patty et al (9), Shrilakshmi 25.25\% and Ejaz Butt et al (10). Women are more likely to develop a meningioma, (5) as in our study, females were more commonly affected 108 cases (54\%) compared to males 92 (46\%). A female preponderance for meningioma correlates with an endogenous hormone level and exogenous hormone replacement in postmenopausal women (in whom an increased incidence of meningioma is seen) as compared with postmenopausal women who have not taken exogenous hormone replacement therapy.

The present study revealed that the incidence of meningioma was common in the age group 41-60 years $48 \%$ of patients. The mean age was 48.54 years. In the studies done by A B Shah et al (7), Shrilakshmi (2), the most common age group involved were also 40-50 years.

Meningiomas in children are less common (11), and in our study, there were only 9 cases of meningiomas in children of age group 11-20 years. The intracranial location of meningiomas were distributed as to be the convexities were commonly involved $40 \%$, in which frontal was more common, $45.45 \%$, followed by the parasagittal and falcine meningioma were $20 \%, 10 \%$ were in sphenoid wing, $11 \%$ in CP angle and posterior fossa. In a study by shrilakshmi et al, $61.11 \%$ of tumours were located in convexity. The clinical presentation of meningiomas, depends on tumour location (12). The symptoms at presentation are rarely precipitous, but often insidious. Onset of slowly evolving headache is common and usually not associated with other symptoms suggestive of raised intracranial pressure, reflecting the slow growth of these tumours. A history of partial seizures is common for convexity meningiomas and an insidious personality change that is confused with dementia or depression is common in patients with large inferior frontal meningiomas (4). In our study, the most common clinical symptoms were headache, seizures and vomiting. The common radiological findings were mass lesions with pressure effect on adjacent structures and peritumoral edema.
Meningiomas divided in wide variety of histological patterns. Our present study revealed that the most common histologic type was meningothelial (38.89\%), similar to studies by Nasrin Samadi et al (13) Sangamithra et al (14), Thomas Backer et al (15), followed by atypical meningiomas (16.67\%). The other variants were fibroblastic (11.11\%), transitional (11.11\%), psammomatous variant, angiomatous, lympho-plasmacytic and fibrous (5.56\%) each. According to WHO (5) atypical meningiomas have more than three of the following features - increased cellularity, smaller cells with high N/C ratio, greater than 4 mitotic figures/ 10HPF, prominent nucleoli and geographic necrosis. In our study (16.67\%) of atypical meningiomas were reported. Singh Avninder et al (16) reported that papillary meningiomas and anaplastic meningiomas are rare and constitute $1-2.5 \%$ of all meningiomas. In the studies done by S Hoon et al (17) and Gottfried et al. (18) Histological analysis reveals that $80-90 \%$ of meningiomas are benign [World Health Organization (WHO) Grade I], 5-15\% are atypical (WHO Grade II) and associated with a marked increase in recurrence. Only $1-3 \%$ of the cases become anaplasic or malignant (WHOGrade III), developing a high tendency to invade brain structures, metastasize, and recur. In our study, $16.67 \%$ of atypical meningioma was observed. Though meningiomas are considered to be benign tumours, recurrence is frequently observed (19). Benign meningiomas can recur following incomplete resection, if large and associated with monosomy 14 and del (1p36). The extent of surgical resection depends on the size of the tumour, site, and its relation to vital structures. The best accepted system for prediction of recurrence is the Simpson grading system for completeness of resection (20), which evaluates the invasion of the venous sinuses, tumour nodules in adjacent dura, and infiltration of unresected bone by meningothelial cells. The recurrence rates that Simpson refers to $9 \%$ for grade I, $16 \%$ for grade II, $29 \%$ for grade III, 39\% for grade IV, and $100 \%$ for grade $V$, respectively.

\section{Simpson's scale of grading divides the extent of resection into 5 grades:}

Grade I: Complete removal

Grade II: Complete removal with coagulation of dural attachment 
Grade III: Complete removal, without coagulation of dural attachment or resection of involved sinus or hyperostotic bone

Grade IV: Subtotal resection

Grade V: Decompression biopsy.

For patients with resection grades IV and V, endpoint for recurrence was enlargement of the remaining tumour, shown on MRI or CT. In addition, histological characteristics of malignancy such as peritumoral brain edema, cellular pleomorphism, nuclear atypia, presence of macronuclei, atypical mitoses, increase of neovascularization, brain invasion and necrosis, favour recurrence rate of meningiomas (20).

The treatment in grade I meningioma is total resection. In grade II and grade III meningiomas (2), surgery and adjuvant radiotherapy are the treatment of choice. Extent of surgical resection is one of the most important factors in predicting recurrence along with histological grading.

Bone flap removal was done for 2 cases due to intraoperative brain swelling. Immediate complication was haematoma in 2 cases (3.84\%), for which reexploration was done. Major post-operative complications in our study were convulsions $21.1 \%$, wound infection $17.3 \%$, CSF leak in $9.62 \%$, meningitis in $11.53 \%$, of cases. All the patients before surgery were adequately treated with anti-convulsive therapy. Postoperatively $15 \%$ of cases developed convulsions within $48 \mathrm{hrs}$ after surgery. They were controlled with increase in the dose of anti-epileptics or addition of another antiepileptic drug. The major morbidity in our series was post-operative infection, in the form of wound infection, CSF leak, and meningitis.

Follow-up period was 6 months to 5 years. Cases were followed up with $\mathrm{CT}$ brain in symptomatic patients. Twelve cases of recurrence are noted on follow-up for which incomplete resection was done. Anyhow follow-up period was not enough to assess the recurrence as meningiomas are slow growing tumours.

\section{REFERENCES}

1. Jose Carlos, Adrian Mosquera. An integrative analysis of meningioma tumors reveal the determinant genes and pathways of malignant transformation. Frontiers in oncology.2014;(4)147.

2. Shrilakshmi.S, Meningiomas: a clinicopathological study. Int J Med Res Health Sci. 2015;4(4):827-831.
3. YangSY, ParkCK, ParkSH, KimDG, ChungYS, JungHW. Atypical and anaplastic meningiomas: prognostic implications of clinicopathological features. J Neurol NeurosurgPsychiatry .2008; 79(5):574-80.

4. Jason rockhill, Maciej mrugala, Marc c. chamberlain, Intracranial meningiomas: an overview of diagnosis and treatment. Neurosurg Focus 2007; 23 (4): E1.

5. Deborah L. Commins, Roscoe D. Atkinson and Margaret E. Burnett, Review of meningioma histopathology; Neurosurg Focus 2007; 23 (4): E3.

6. A Perry, D. N. Louis, B. W. Scheithauer. H. Budka, A. von Deimling: Meningiomas in WHO Classification of Tumours of the Central Nervous System, 4th Edition, IARC press, Lyon 2007; 1:164-72.

7. AB Shah, GA Muzumdar, AR Chitale. Meningiomas: A Report of a hospital-based registry. Indian J Pathology and Microbiology 2005; 48(4): 468-71.

8. Ruberti R F, The surgery of Meningiomas: A review of 215 cases. African Journal of Neurological Sciences 2007.

9. Intisar S.H Patty. Central Nervous System Tumours- A Clinicopathological study. J Dohuk Univ.2008; 11, (1):17380.

10. M. Ejaz Butt, Saeed A. Khan, Naseer A. Chaudrhy, G.R. Qureshi. Intra-Cranial space- occupying lesions- A morphological analysis. Biomedica 2005; 21.31-35.

11. Nirav Mehta, SanatBhagwati, and GeetaParulekar; Meningiomas in children: A study of 18 Cases; J Pediatric Neurosciences 2009;4(2): 61-65.

12. Rutten I, Cabay JE, Withofs N, Lemaire C, Aerts J, Baart V, et al: PET/CT of skull base meningiomas using 2-18F-fluoro-L tyrosine: initial report. J Nucl Med 48:720-5, 2007.

13. Nasrin Samadi, Seyed Ali Ahmadi; Meningioma: A clinicopathological evaluation. Malaysian Journal of Medical sciences 2007;14(1): 46-52.

14. Sashidhar Babu, Shantveer G Uppin, Megha S Uppin, et al. Meningiomas: Correlation of Ki67 with histological grade. Neurology India 2011; 59 (2) 204- 07.

15. Jan Regelsberger, Christian Hagel, PedramEmami, Thorsten Ries, Oliver Heese, Manfred Westphal; Secretory meningiomas: A benign subgroup causing life-threatening complications; Neuro Oncology 2009 December; 11(6): 819-24.

16. Singh Avninder, SarjotVermani, Sharma Shruti et al. Papillary meningioma: A rare but distinct variant of malignant meningioma. Diagnostic Pathology 2007; 2:3.

17. Sang HoonYoon, Chun Kee Chung, and Tae AhnJahng.Surgical Outcome of Spinal Canal Meningiomas.J Korean NeurosurgSoc. 2007; 42(4): 300-04.

18. Oren N. Gottfried, Wayne Gluf, Alfredo Quinones- Hinojosa, Peter Kan, and Meic H. Schmidt.Spinal meningiomas: Surgical management and outcome Neurosurg Focus 2003; 14 (6): 2.

19. Konstantinos Violaris, Vasileios Katsarides, Maria Karakyriou, Pavlos Sakellariou, Surgical Outcome of Treating Grades II and III Meningiomas:A Report of 32 Cases.Neuroscience Journal 2013; 706481, 4 pages.Konstantinos Violaris, Vasileios Katsarides, Pavlos 
Sakellariou, The Recurrence Rate in Meningiomas: Analysis of Tumor Location, Histological Grading, and Extent of
Resection. Open Journal of Modern Neurosurgery, 2012; 2, 6-10 